\title{
Was ist ein Hindernis? - Fachliche Aushandlungen im Sachunterricht am Beispiel der Mobilitätsbildung
}

\author{
Nina Skorsetz $\cdot$ Marina Bonanati $\cdot$ Diemut Kucharz
}

Eingegangen: 1. Juni 2020 / Angenommen: 11. Oktober 2020 / Online publiziert: 25. Januar 2021

(C) Der/die Autor(en) 2021

Zusammenfassung Anhand eines Transkripts aus dem Sachunterricht an einer inklusiven Grundschule wird in diesem Beitrag herausgearbeitet, was Fachlichkeit am Beispiel der Radfahrausbildung in einer vierten Klasse ausmacht. Für die Videographien im Sachunterricht wurden Schulen ausgewählt, die sich selbst als inklusiv beschreiben. So beschreiben sie beispielsweise, dass der Anspruch auf sonderpädagogische Förderung erst im Übergang zur weiterführenden Schule festgestellt wird. Untersucht wird, wie die Teilnehmenden in einer Unterrichtsstunde ihre Vorstellungen zu dem fachlichen Begriff Hindernis aushandeln. Es zeigt sich, dass bei der Bearbeitung des Themas Vorbeifahren an einem Hindernis im Material kein Rückgriff auf die begrifflichen Vorstellungen bzw. Konzepte der Kinder vorgesehen ist. Da die Lernenden dennoch einen Aushandlungsprozess initiieren, in den sie auch die Lehrkraft einbeziehen, ist eine Auseinandersetzung mit den kindlichen und fachlichen Vorstellungen zu beobachten.

Schlüsselwörter Inklusion · Schülervorstellungen · (Prä-)Konzepte · Rekonstruktive Unterrichtsforschung $\cdot$ Fachlichkeit $\cdot$ Mobilitätsbildung

Dr. N. Skorsetz $(\bowtie) \cdot$ Dr. M. Bonanati

Fachbereich Erziehungswissenschaften 04, Institut für Pädagogik der Elementar- und Primarstufe, Goethe Universität Frankfurt am Main, Theodor-W.-Adorno-Platz 6, 60629 Frankfurt, Deutschland E-Mail: skorsetz@em.uni-frankfurt.de

Dr. M. Bonanati

E-Mail: bonanati@em.uni-frankfurt.de

Prof. Dr. D. Kucharz

Fachbereich Erziehungswissenschaften 04, Institut für Pädagogik der Elementar- und Primarstufe, Professur für Grundschulpädagogik mit dem Schwerpunkt Sachunterricht, Goethe Universität Frankfurt am Main, Theodor-W.-Adorno-Platz 6, 60629 Frankfurt, Deutschland E-Mail: kucharz@em.uni-frankfurt.de 


\title{
What is an obstacle?-Specialist negotiations in social studies lessons using the example of mobility education
}

\begin{abstract}
In Germany, children usually participate in a cycling training in the fourth class. Based on a transcript from a social and science studies lesson in an inclusive school on the topic of driving past an obstacle we analyzed how the participants negotiate their ideas on the technical term obstacle. The analysis showed that the question of what an obstacle is was not intended by the teaching materials to refer to the children's conceptual ideas or concepts. We observed that the learners nevertheless initiate a negotiation process about their own and professional concepts in which they also involve the teacher.
\end{abstract}

Keywords Inclusion -(Pre-)concepts - Reconstructive classroom research · Professionalism $\cdot$ Mobility education

\section{Einleitung}

Mobilität ist eine wichtige Voraussetzung für die soziale Teilhabe aller Menschen. Barrierefreie Mobilität ist vor dem Hintergrund der UN-Behindertenrechtskonvention (vgl. UN-BRK 2006) für eine inklusive Gesellschaft evident, aber noch längst nicht erreicht. Auch für Kinder spielt Mobilität für ihre soziale Teilhabe eine bedeutende Rolle, verstärkt durch veränderte Freizeit- und Bewegungsmöglichkeiten (z. B. Günther und Degener 2009). Deshalb ist Mobilitätsbildung ein Bestandteil grundlegender Bildung und findet traditioneller Weise im Sachunterricht der Grundschule statt.

Anhand der Mobilitätsbildung soll exemplarisch deutlich werden, wie Fachlichkeit im Sachunterricht im Spannungsfeld zwischen lebensweltlichen Erfahrungen und fachlichen, abstrakten Begriffen konstruiert wird. Vor dem Hintergrund eines übergeordneten Inklusionsanspruchs wird zudem die Frage nach der Teilhabe der Kinder an diesem Konstruktionsprozess relevant.

Im Rahmen der folgenden Darstellung erfolgt neben der Betrachtung der aktuellen fachdidaktischen Diskussions- und Forschungslage zur Verkehrserziehung im Rahmen einer umfassenden Mobilitätsbildung zunächst eine Auseinandersetzung mit Fachlichkeit im inklusiven Sachunterricht.

Für die anschließende Analyse dienen Ausschnitte aus Videographien einer Sachunterrichtstunde als Datengrundlage, die in einer heterogenen Lerngruppe an einer Grundschule mit inklusivem Selbstverständnis im Rahmen der Verkehrserziehung erhoben wurden. Auf der Mikroebene wird durch detaillierte Interaktionsanalysen rekonstruiert, wie die Teilnehmenden in dieser Unterrichtsstunde ihre Vorstellungen zu einem fachlichen Begriff aushandeln. Die Ergebnisse dieser Rekonstruktion werden schließlich vor dem Hintergrund der fachdidaktischen Diskussion reflektiert. 
Abb. 1 Das doppelte didaktische Dreieck des Sachunterrichts (nach Thomas 2015; Pech 2009)
Kind

Welt

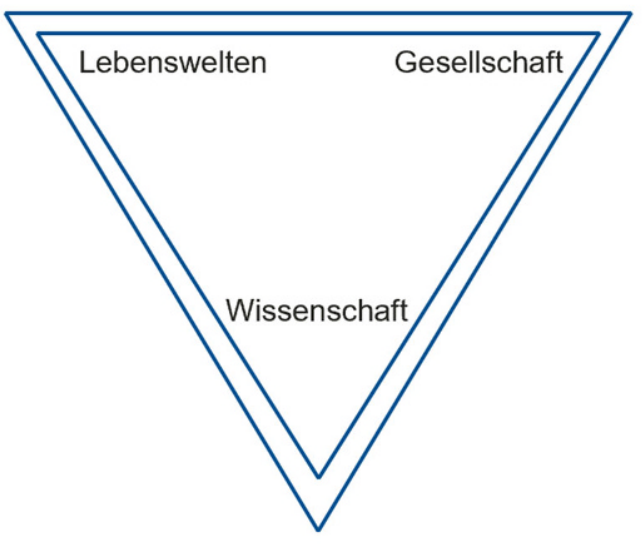

Sache

\section{Fachliches und begriffliches Lernen im Sachunterricht}

\subsection{Das Schulfach Sachunterricht und seine Fachlichkeit}

Sachunterricht hat die Aufgabe und das Ziel, Kinder bei der Welterkundung, bei der Erschließung ihrer Lebenswelt zu unterstützen. Das geschieht, indem Phänomene, Sachverhalte und die kindlichen Fragen an ihre Welt aufgegriffen werden (Köhnlein 2015). Damit besteht die besondere Herausforderung des Faches darin, sich in dem Spannungsfeld zwischen den beteiligten Fachwissenschaften, den gesellschaftlichen Forderungen und dem Kind als Lernendem zu verorten (Thomas 2015). Diese Bezugsgrößen lassen sich nach Pech (2009) in ihrem Verhältnis zueinander als doppeltes didaktisches Dreieck darstellen (s. Abb. 1). Insofern wird die Frage nach der Fachlichkeit im Sachunterricht weniger von den Bezugswissenschaften als vom Thema ausgehend und seiner Verortung in diesem Spannungsfeld bestimmt.

Der Bildungsgehalt dieser so gewonnenen Themen bzw. Gegenstände des Sachunterrichts ergibt sich also nicht aus der Logik einzelner Fachdisziplinen, sondern die verschiedenen Fachperspektiven werden herangezogen, um diese Fragen, Phänomene etc. vielperspektivisch zu klären. Dabei bedient man sich einerseits der fachtypischen Methoden ${ }^{1}$ als auch der Inhaltsaspekte, die fachspezifisch geklärt werden können. Bei der Klärung geht es um eine Versachlichung und Weiterentwicklung der kindlichen Vorstellungen von der Welt (Kahlert 2009).

\subsection{Fachlichkeit in der inklusiven Unterrichtsforschung}

Inklusiver Fachunterricht gilt in Deutschland als noch wenig beforscht (Hackbarth und Martens 2018). Dieses Forschungsdesiderat wird auch für den inklusiven Sachunterricht konstatiert (Pech et al. 2018, 2019). Vereinzelt liegen frühe empirische

\footnotetext{
1 auch Denk-, Arbeits- und Handlungsweisen genannt (GDSU 2013).
} 
Studien vor (Seitz 2005; Schomaker 2007), dennoch hat sich bislang trotz steigender Publikationen keine systematische inklusive Sachunterrichtsforschung etabliert (Pech et al. 2018). Hier stellt sich die Frage, ob es überhaupt einer spezifischen inklusiven Didaktik für den Sachunterricht bedarf (Kucharz 2015; Pech und Rauterberg 2016), oder ob die Konzeption der Vielperspektivität als anschlussfähig für Inklusion betrachtet werden kann (s. a. Hinz 2011; Simon 2017), da sie vielfältige Zugänge für heterogene Lerngruppen bietet (GDSU 2013), die auch für inklusive Settings geeignet sind. Bereits in den 1980er-Jahren forderte Feuser (2018 [1984]) für den integrativen Unterricht das Lernen am gemeinsamen Gegenstand, für das Formen des differenzierten Unterrichts zur Anwendung kommen, der die individuellen Lernvoraussetzungen der heterogenen Kindergruppe berücksichtigt (Kucharz 2015).

\subsection{Konzepte und Bedeutung von Fachbegriffen}

Im fachdidaktischen Diskurs - vor allem im Bereich der Naturwissenschaften und des Sachunterrichts (Adamina et al. 2018) - wird die Bedeutsamkeit von Schüler*innenvorstellungen und deren Weiterentwicklung diskutiert. Mit Vorstellungen sind ganz allgemein ,unterschiedlich ausgeprägte Bewusstseinsinhalte“ (Möller 2018, S. 35) von Lernenden gemeint, die sich nicht nur auf fachliche Inhalte, sondern auch auf Erfahrungen und Erlebnisse beziehen können. Vorstellungen werden sowohl zu konkret Anschaulichem, wie Phänomenen, als auch zu abstrakten Konstrukten und Begriffen entwickelt. Werden diese Vorstellungen im Unterricht genutzt, spricht Möller (ebd.) von Vorwissen oder Präkonzepten, die im Rahmen des Lernprozesses weiterentwickelt werden sollen. Präkonzepte repräsentieren damit eine Art fachliche Ausgangslage, die meist eher lebensweltlich konturiert ist und auf die die Lernenden rekurrieren, wenn sie mit weiteren Wissensinhalten oder Phänomenen konfrontiert werden, und enthüllen somit, warum Kinder z. B. in einer bestimmten Weise argumentieren. Dabei können diese Vorstellungen bereits theorieähnlich strukturiert sein oder aus unzusammenhängenden Wissensbeständen bestehen (Adamina et al. 2018). Es hat sich gezeigt, dass Vorstellungen nur schwer veränderbar sind und ein radikaler Konzeptwechsel durch Unterricht kaum möglich ist (ebd.). Möller (1999) konnte auch zeigen, dass Unterricht, der nicht auf das Vorwissen der Kinder eingeht, nur so genanntes träges Wissen hervorbringt, das nicht dazu beiträgt Schüler*innenvorstellungen weiterzuentwickeln.

Im aktuellen Forschungsdiskurs wird der Begriff Schüler*innenvorstellungen eher dann genutzt, wenn Vorwissen und Erfahrungen rekonstruiert werden sollen. Im naturwissenschaftlichen Bereich ${ }^{2}$ der Conceptual Change Forschung wird davon gesprochen, unwissenschaftliche Präkonzepte durch Unterricht oder andere Interventionen in eher wissenschaftlich belastbare Postkonzepte weiter zu entwickeln (vgl. Möller 2018). Für den Bereich des Phänomens Schwimmen und Sinken und andere Beispiele wurden hier bereits Stufenmodelle der Vorstellungsveränderungen entwickelt (Jonen et al. 2003). Die Präkonzepte sollen von der Lehrkraft zu Beginn

\footnotetext{
2 Auch im gesellschaftswissenschaftlichen Bereich bringen Lernende bereits Vorstellungen mit - siehe dazu beispielsweise die Untersuchung von Baar und Maier (2012) zu Präkonzepten zu dem Begriff Familie.
} 
einer Unterrichtseinheit erhoben werden, um daran im Unterricht anzuknüpfen, deren Grenzen ggf. deutlich zu machen und so eine Weiterentwicklung anzubahnen. Wenn dies sprachlich geschieht, spielen außer den kognitiven auch die verbalen Fähigkeiten der Kinder eine Rolle, um die eigenen Vorstellungen z.B. zu einem Fachbegriff zum Ausdruck bringen zu können (Gropengießer 2001).

\subsection{Fachliches Lernen in der Mobilitätsbildung}

Dass Mobilität für Kinder und für Menschen mit und ohne Beeinträchtigung eine große Bedeutung für ihre gesellschaftliche Teilhabe hat, wurde bereits in der Einleitung ausgeführt. Im Sinne einer Unfallprävention vor allem seit den 1970er-Jahren stand das Erlernen von Verkehrsregeln und Verhaltensweisen im Fokus (Spitta 2015). Die Mobilitätsbildung im vielperspektivischen Sachunterricht geht über Verkehrserziehung hinaus. Ziele der „Mobilitätsbildung in einer urbanisierten Welt“ (Kaiser 2016, S. 189) sind ,eine selbstständige, reflektierte, umwelt- und verantwortungsbewusste Wahl der benutzten Verkehrsmittel zu treffen sowie, in Bezug auf Mobilität im Kontext der Bildung für nachhaltige Entwicklung, an gesellschaftlichen Problemlösungen im eigenen Wohn- und Schulumfeld mitzuwirken“ (GDSU 2013, S. 74; s. auch Schneider 2018; KMK 2012).

In dem hier analysierten Sachunterricht wird allerdings Verkehrserziehung im engeren Sinne realisiert, indem die Kinder auf die anstehende Fahrradprüfung vorbereitet werden. Die Fahrradprüfung ist das gemeinsame Ziel im inklusiven Sachunterricht und ist fachlich vom gesellschaftlichen Interesse an der Verkehrstüchtigkeit der Kinder dominiert. Bei diesem Gegenstand wird aus gesellschaftlicher Perspektive nicht in Frage gestellt, ob er für alle Kinder relevant ist. Es gibt kaum konkrete Anregungen, wie Lehrkräfte - entsprechend unterschiedlicher Voraussetzungen Verkehrserziehung didaktisch aufbereiten können. Auf der Medienseite der Verkehrswacht finden sich dazu folgende Äußerungen:

„Die ,Verkehrserziehung für Menschen mit einer Behinderung' gibt es nicht. Unterschiedliche Mobilitätsbeeinträchtigungen erfordern unterschiedliche Förderschwerpunkte. Im Ansatz unterscheidet sich Verkehrserziehung für Heranwachsende mit einem Handicap aber kaum von der allgemeinen schulischen Mobilitätsbildung. Die Radfahrausbildung ist auch dort etabliert. Wir empfehlen, jedes Kind, soweit dies möglich ist, an der Ausbildung und an der Lernkontrolle teilnehmen zu lassen. Bei der Beurteilung sind die individuellen Voraussetzungen der Schüler zu berücksichtigen“" (Verkehrswacht o.J.).

Aus- und Fortbildungsangebote für Lehrkräfte in diesem Bereich sind selten, so dass sich eine vielperspektivische Sicht auf das Thema im Sinne einer Mobilitätsbildung nur schwer durchsetzen kann (Spitta 2015). In Bezug auf die bundesweit durchgeführte Radfahrausbildung im vierten Schuljahr konstatiert Schneider (2018), dass der Fokus häufig nicht auf Mobilitätsbildung, sondern nur auf dem Erlernen von Verkehrs- und Sicherheitsregeln sowie dem richtigen Umgang mit dem Fahrrad liege, und häufig außerschulische Lernangebote von Polizei und Verkehrswacht genutzt würden, die eher aus Einweisungsprogrammen mit reiner Vermittlungsabsicht bestünden. Auch Funk et al. (2013), die Experteninterviews und eine Fragebogenbefragung bei Fach- und Lehrkräften in Kindergarten und Grundschule $(n=1920)$ 
durchführten, fanden heraus, dass die Verkehrserziehung zwar kein Randthema darstelle, der Schwerpunkt aber - im Gegensatz zu der geforderten umfänglichen Mobilitätsbildung - auf der Sicherheitserziehung und spielerischen Übungen zur Verkehrsvorbereitung liege.

Günther und Degener (2009) konnten in ihrer Befragung bei rund 1000 Verkehrserziehungs-Dienststellen und Schulen zeigen, dass diese zwar durch häufigeres Fahrradfahren im Straßenverkehr praxisnäher geworden sei, jedoch noch immer konkrete Handlungsdispositionen wie Anfahren etc. im Vordergrund stünden.

Abschließend lässt sich konstatieren, dass die Mobilitätsbildung im Allgemeinen sowie die Radfahrausbildung im Speziellen kaum eine Rolle in der Sachunterrichtsforschung spielen (Schneider 2018). Fachliches Lernen bezieht sich in den wenigen vorliegenden empirischen Studien immer auf die Vermittlung von spezifischem Wissen, wie z.B. der Straßenverkehrsordnung (StVO), sowie praktischem Können, wie Fahrradfahren (ebd., S. 22).

Auch im vorliegenden Beispiel stehen diese Inhalte im Vordergrund. Als fachlicher Bezug steht in dem Unterricht ein Arbeitsheft der Verkehrswacht im Zentrum. Die Analysen zeigen, dass für die Kinder darüber hinaus begriffliche Klärungen von Bedeutung sind: Der Begriff Hindernis taucht in dem von uns untersuchten Sachunterricht im Rahmen der Radfahrausbildung auf und wird für die Kinder zu einem zu verhandelnden Gegenstand. Er rekurriert als Fachbegriff auf den Kontext des Straßenverkehrs und wird in dem zu bearbeitenden Heft offenbar als bekannt vorausgesetzt. In der StVO wird unter $§ 6$ Vorbeifahren folgendes definiert: „Wer an einer Fahrbahnverengung, einem Hindernis auf der Fahrbahn oder einem haltenden Fahrzeug links vorbeifahren will, muss entgegenkommende Fahrzeuge durchfahren lassen. Satz 1 gilt nicht, wenn der Vorrang durch Verkehrszeichen anders geregelt ist. Muss ausgeschert werden, ist auf den nachfolgenden Verkehr zu achten und das Ausscheren sowie das Wiedereinordnen - wie beim Überholen - anzukündigen“ (StVO § 6). Eine genauere Erläuterung, was ein Hindernis im Straßenverkehr darstellt, findet sich hier nicht. Im Wörterbuch der deutschen Sprache werden drei Bedeutungen aufgeführt (DWDS o.J.):

1. hindernder Umstand, Sachverhalt; Hemmnis, Schwierigkeit

2. etwas, was das direkte Erreichen eines Ziels, das Weiterkommen be- oder verhindert

3. auf einer (Renn-)Strecke aufgebaute Vorrichtung, die beispielsweise von Sportler*innen oder Pferden überwunden werden muss

Es zeigt sich also, dass der Begriff unterschiedliche, auch metaphorische Bedeutungen haben kann. Der Straßenverkehrsordnung liegt vermutlich die zweite Bedeutung zugrunde. Es ist anzunehmen, dass die Vorstellungen der Kinder zum Begriff Hindernis eher lebensweltorientiert und von ihren konkreten Erfahrungen geleitet sind. 


\section{Methodische Überlegungen}

Mit der übergreifenden Frage danach, was im Sachunterricht wie zum Gegenstand gemacht wird, fokussieren wir fachlich-inhaltliche Aushandlungen. Bräuer et al. (2018) verorten unterschiedliche Forschungsansätze, die sich für Fachlichkeit interessieren, entlang einer Topologie zweier Dimensionen. Dimension eins bezieht sich auf die Vorstellung von Fachlichkeit und Dimension zwei auf die Normen der Qualität fachlicher Vermittlungs- und Aneignungsprozesse; beide bewegen sich zwischen Setzen und Rekonstruktion. Im Sinne dieser Topologie betrachten wir fachliche Aushandlungen im Unterricht auf Dimension eins zwischen dem Setzen von Fachlichkeit als substanzieller Vorstellung und der Rekonstruktion von Fachlichkeit im Sinne einer sozialen Konstruktion. Dies bedeutet, dass wir zum einen - unter Bezugnahme auf eine interaktionstheoretische Perspektive - rekonstruieren, wie Lehrperson und Schüler*innen in der sozialen Situation Unterricht fachliche Aushandlungen hervorbringen. Zum anderen werden Ansätze der fachdidaktischen Diskussion den Analysen gegenübergestellt, so dass ein erweiterter Blick auf Fachlichkeit möglich wird.

Zentrales methodisches Verfahren zur Analyse von Unterrichtstranskripten ist die Interaktionsanalyse im Sinne der interpretativen Unterrichtsforschung (Breidenstein 2002). Fachlich-inhaltliche Interaktionen werden in einem ersten Schritt identifiziert, indem die Unterrichtsvideos segmentiert und die didaktische Gestaltung des Lernsettings zusammengefasst wird. Anschließend erfolgt die detaillierte Sequenzanalyse (in Anlehnung an die Analysemaximen nach Krummheuer und Naujok 1999). Interaktionsanalysen zielen ,,[...] auf eine empirische Analyse von Interaktionsprozessen im Sinne eines von mehreren anwesenden Personen in wechselseitiger Abhängigkeit erzeugten Prozesse[es]“" (Krummheuer und Brandt 2001, S. 14; Herv. i. O). Aus diesem Wechselprozess aufeinander bezogener Rede- und Handlungszüge ergibt sich die thematische Entwicklung der Interaktion inklusive Bedeutungsaushandlungen (Krummheuer 2012).

Für die hier analysierte Szene wurden Sachunterrichtsstunden einer vierten Klasse an einer Grundschule im Rhein-Main-Gebiet videografiert. Neben Aufnahmen aus zwei Totalen wurden feststehende Kameras vor jeden Gruppentisch in der Klasse positioniert, so dass die Interaktionen der Schüler*innen dokumentiert werden konnten. Durch Audiorekorder auf den Gruppentischen sowie einem Mikrofon für die Lehrperson konnten alle Gespräche festgehalten werden.

Analyseleitend für die nachfolgend präsentierten Szenen sind die Fragen: Wie handeln die Teilnehmenden in einer Unterrichtsstunde im Rahmen der Radfahrausbildung aus, was Hindernisse sein können? Welche Bedeutung haben für diesen Ko-Konstruktionsprozess die Vorstellungen der Schüler*innen, die Äußerungen der Lehrerin sowie die verwendeten Materialien? 


\section{Ergebnisse der Interaktionsanalyse - Was können Hindernisse sein?}

\subsection{Zum Kontext der Szene}

Das Transkript dokumentiert die Handlungen von vier Jungen (Edgar, Piet, Gerald, Sujan) $)^{3}$ an einem Gruppentisch in einer Sachunterrichtsstunde in einem vierten Schuljahr. Die Klasse beschäftigt sich seit ca. zwei Wochen mit der Radfahrausbildung, an deren Ende nach einem Vierteljahr Vorbereitungszeit die Radfahrprüfung stehen soll. Im Zentrum steht von der Verkehrswacht zur Verfügung gestelltes Arbeitsmaterial zur Verkehrserziehung (Verkehrswacht 2019).

In einem schon in vorhergehenden Stunden genutzten DINA 4 Arbeitsheft (ebd., S. 26) sollen zwei Seiten bearbeitet werden. Auf der linken Seite sind oben zwei Aufgabenstellungen formuliert: 1. Was können Hindernisse sein? Zähle auf! 2. Welche Verkehrsschilder zeigen dir Hindernisse an? Die freien Flächen darunter fordern dazu auf, schriftlich zu antworten. Des Weiteren sollen von den Kindern Figuren zu den zu befolgenden Schritten ausgeschnitten und in der richtigen Reihenfolge aufgeklebt werden. Auf der Rückseite des Arbeitsheftes sind zahlreiche Verkehrsschilder abgebildet, auf die die Schüler*innen zur Beantwortung der zweiten Frage zurückgreifen.

Die als Stationenarbeit angelegte Beschäftigung mit dem Vorbeifahren an einem Hindernis gliedert sich in lehrergelenkte Phasen und selbstständige Gruppenarbeitsphasen zur eigenständigen Erarbeitung der Aufgaben im Arbeitsheft. Die detaillierte Sequenzanalyse von vier Szenen aus dem Verlauf der Gruppenarbeit zeigt, wie die Schüler Piet, Gerald und Edgar die Frage aushandeln, was Hindernisse sein können. Die Aushandlungen der Jungen pendeln dabei zwischen individuellen Lebensweltbezügen (Beispiele 1, 3, 4) und den Vorgaben des Arbeitsmaterials durch Aufgabenstellungen und Abbildungen (Beispiele 2, 4).

Szene 1: Können Steine ein Hindernis sein? (Min. 23:00-23:28)

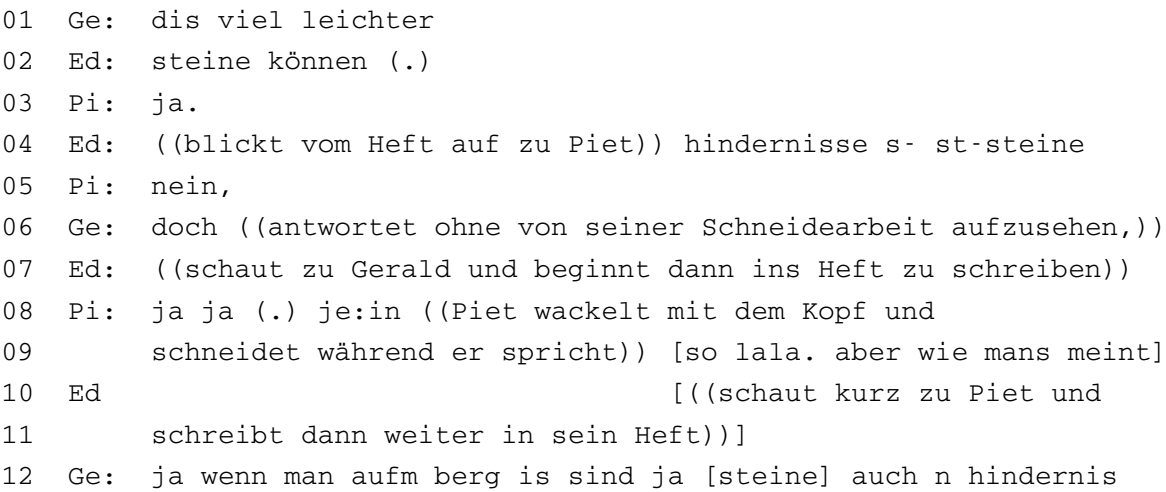

\footnotetext{
${ }^{3}$ Die Namen der Jungen wurden pseudonymisiert. Die in dieser Szene vornehmlich nonverbalen Handlungen von Sujan werden dann in das Transkript aufgenommen, wenn sie auf die anderen Jungen ausgerichtet sind. Er arbeitet in einem anderen Arbeitsheft.
} 
Die Äußerung des Schülers Edgar in Zeile 2 und 4 bildet eine Antwort auf die im Arbeitsheft formulierte Frage, was ein Hindernis sein kann. Die Frage setzt damit schüler*innenseitig eine Vorstellung von Hindernis voraus und fordert eher einen theoretisch-kognitiven Zugang, indem Hindernisse aufgezählt werden sollen. Schüler*innenerfahrungen und -vorstellungen von Hindernissen sollen nicht ausgehandelt werden.

Edgars Beitrag (Z. 4) initiiert eine Aushandlung zwischen Piet und Gerald. Piet widerspricht Edgar (,nein“), woraufhin Gerald sich mit ,doch“ einbringt und somit Edgars Einschätzung zustimmt. Edgar reagiert nonverbal, indem er zu ihm schaut, dann aber (weiter) schreibt. Mit seinem nächsten Beitrag bestätigt Piet zunächst ,ja, ja“, relativiert dann aber durch drei Formulierungen (,jein“, ,,so lala“ und ,wie mans meint"), die weder Zustimmung noch Verneinung ausdrücken und seine Position somit vage bleibt. Gerald formuliert nun ein Argument (Z. 12), womit er sein ,doch“ unterstreicht. Er konstruiert eine Situation an einem bestimmten Ort (,aufm Berg“"), an dem Steine ein Hindernis seien. Piet setzt einen anderen Ort dagegen (Z. 14) „auf so einer Straße“ „, bei der Karibik“, womit er den Kontext Verkehr aktiviert und deutlich macht, dass es kontextabhängig ist, ob ein Stein ein Hindernis darstellt.

In diesem Abschnitt bilden subjektive Lebensweltbezüge und Vorstellungen der Schüler die Grundlage für ihre Aushandlung. Die Aushandlungen sind lebensweltlich orientiert, da dieses Beispiel ${ }^{4}$ für ein Hindernis der Vorstellung der Schüler davon entspringt, was sie auf einem Weg behindern könnte.

Szene 2: Kann eine Kurve ein Hindernis sein (I)? (Min. 24:46-24:53)

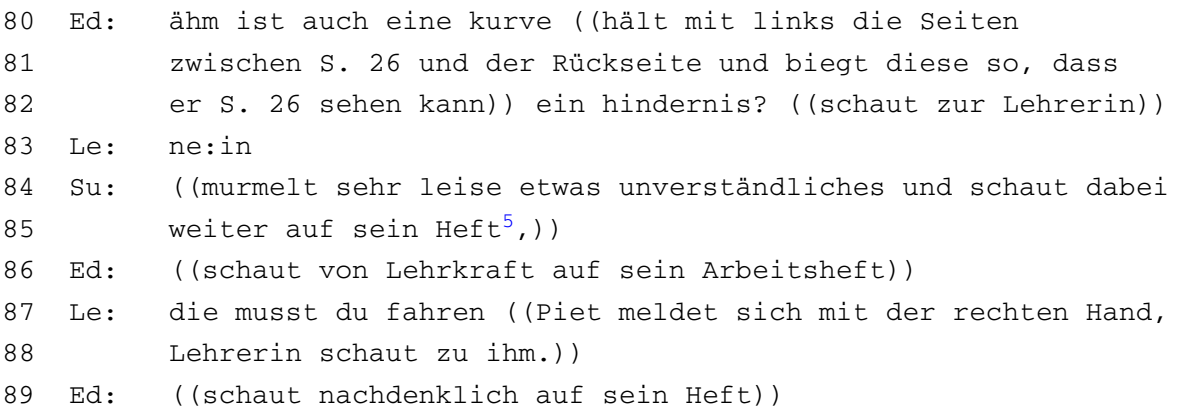

Da Edgar zur Rückseite des Arbeitsheftes mit den Verkehrsschildern blättert, ist anzunehmen, dass das dort abgebildete Schild Kurve Auslöser für seine Frage ist, ob auch eine Kurve ein Hindernis sei (Z. 80/82). Die Konjunktion ,auch“ verweist

\footnotetext{
4 Weitere Beispiele sind: tote Tiere auf der Fahrbahn, Bäume, Hundekot.

5 Zwischenzeitlich arbeitet er, nach einer Besprechung mit der Lehrerin, ebenfalls in dem Arbeitsheft. Im Verlauf der folgenden Szenen ist er damit beschäftigt, die Figuren auszuschneiden.
} 
darauf, dass bereits (innerhalb der Gruppe validierte) Hindernisse gefunden wurden. Außerdem zeigt es an, dass mehrere Lösungen oder eine vollständige Liste gesucht werden, und reagiert damit auf die Aufforderung ,zähle auf". Hinter Edgars Frage, ob eine Kurve auch ein Hindernis sei, könnte seine Vorstellung stehen, das Hindernis all das beschreibt, was ein normales Weiterfahren behindert. Mit dem Bezug auf die Übersicht der Verkehrsschilder, der an mehreren Gruppentischen zu beobachten ist, vermischen die Schüler*innen die Fragen eins und zwei im Arbeitsheft miteinander.

Der Schüler nutzt die Anwesenheit der Lehrerin (Le) in der Nähe des Gruppentisches, um seine Frage an sie zu adressieren. Damit positioniert er sie als Wissensträgerin und als notwendiges Korrektiv, um die Liste der Hindernisse abzusichern. Die Lehrerin nimmt diese Positionierung an, indem sie die Frage verneint (Z. 83). Auf das für die Transkription unverständliche Murmeln des Schülers Sujan an dieser Stelle (Z. 84) erfolgt keine Reaktion. In Zeile 87 ergänzt die Lehrerin ihre Verneinung mit einer Begründung: „die (gemeint ist die Kurve) musst du fahren“, warum sie eine Kurve nicht als Hindernis definiert. Ihre Begründung verweist auf ein Verständnis, dass das Weiterkommen mit dem Fahrrad durch ein Hindernis beeinträchtigt würde. Durch ein Hindernis wird die Normalvorstellung vom Weg verändert. Eine Kurve bildet nach Aussage der Lehrerin keine Irritation dieser, sondern ist Teil der Fahrbahn und muss auf dem Normalweg bewältigt werden. Diese Begründung verweist auch darauf, dass Schilder zu einem bestimmten Verhalten aufrufen. Die Handlungsnorm bei einer Kurve formuliert die Lehrerin so: du musst fahren. Die Vorstellung des Schülers von Hindernis oder die besondere Vorsicht, zu der das Achtung-Zeichen aufruft und die den Schüler*innen bewusst zu sein scheint, thematisiert sie nicht.

Wie ihr Zusatz von dem Schüler verstanden wird, bleibt zunächst offen, da die Lehrerin sich unmittelbar dem nächsten Schüler zuwendet und Edgar nicht mehr verbal reagiert (Z. 89; in Szene 4 wird die Frage erneut aufgeworfen.). Er wird als nachdenklich beschrieben. Auch durch die Aufzählungslogik entsteht der Eindruck, dass das, was als Hindernis gilt, nicht zur Diskussion gestellt, sondern die Definition von Hindernis vorausgesetzt wird. Der Kontext bleibt im Vergleich zur ersten Szene ausgeblendet.

Nachfolgend (ohne Transkript) wiederholt sich das obenstehende Interaktionsmuster (im Sinne eines Frage-Antwort-Spiels): Die Lehrerin veri- oder falsifiziert für zwei Beispiele (einseitig verengte Fahrbahn; Radverkehr) die Kategorisierung nicht eindeutig, betont aber die Bedeutung der Verkehrsschilder und zu welchem Handeln das Schild aufruft. So verweist sie beispielsweise auf eine besondere Aufmerksamkeit, die in der Situation gefordert ist. Es wird u. a. deutlich, dass Warnschilder auf Gefahren hindeuten, die beispielsweise durch ein Hindernis verursacht werden können. Und: auch eine spezifische Straßenführung kann dazu aufrufen, sich ähnlich zu verhalten, wie es ein Hindernis verlangt (,sodass du einen bo:gen darum machen musst“), wie sie es im späteren Plenumsgespräch anführt.

Szene 3: Kann ein Baum ein Hindernis sein? (Min. 27:59-28:15)

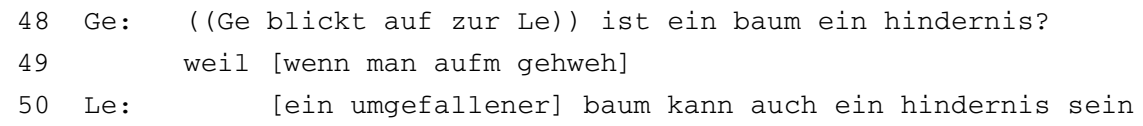




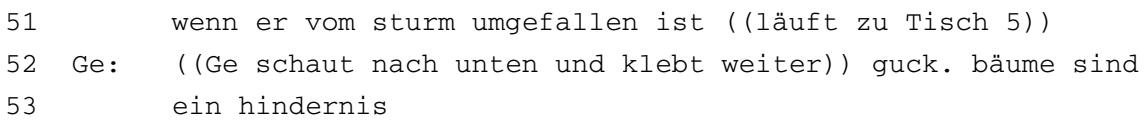

Nun integriert Gerald sich in die Suche nach einer richtigen Lösung (Z. 48). Er stellt seine Frage ähnlich wie Edgar, ergänzt dann aber eine Begründung: ,weil [wenn man aufm gehweh]". Während Edgar zuvor den Namen des Verkehrsschilds nennt und damit auf formale Regelungen der StVO rekurriert, verweist Geralds Formulierung auf eine spezifische Situation (auf dem Gehweg). Seine Begründung wird jedoch von der Lehrerin unterbrochen. Sie bestätigt, dass ein Baum ein Hindernis sein kann - unter der Bedingung, dass er umgefallen ist. Als Ursache dafür, dass der Baum nicht mehr an seiner ursprünglichen Position steht (an der er kein Hindernis sein würde), führt sie einen Sturm an. In diesem Beispiel bildet die Kontextualisierung - die spezifische Position des Baumes - die notwendige Voraussetzung dafür, dass ein Objekt ein Hindernis darstellt.

Gerald widmet sich wieder seiner bisherigen Klebeaufgabe und adressiert dann vermutlich Edgar. Er macht ihn mit dem Imperativ „guck“ darauf aufmerksam, dass seine Idee korrekt ist, indem er wiederholt „bäume sind ein hindernis“. Die Kontextualisierung fällt bei dieser verallgemeinernden (Verwendung des Plurals in Z. 47) Wiederholung weg. Dabei hatte Gerald aus einer lebensweltlichen Perspektive argumentiert, dass Bäume auf dem Gehweg ein Hindernis sein können, was auch für stehende Bäume gelten kann. Seine Erfahrungen sind durch seine sich bisher auf den Gehweg beschränkende Mobilität geprägt. Die Lehrerin dagegen argumentiert aus der Perspektive eines Verkehrsteilnehmers auf der Straße, also im Auto oder auf dem Fahrrad, aus der lediglich umgefallene Bäume ein Hindernis darstellen. Es wird deutlich, dass mit der Erweiterung der Mobilität durch die Radfahrprüfung eine Erweiterung der Perspektiven gefordert ist (vom Gehweg auf die Straße), die den Teilnehmenden hier nicht präsent zu sein scheint.

Szene 4: Kann eine Kurve ein Hindernis sein (II) (Min. 29:04-30:06)

Beispiel 4 zeigt, wie die Jungen das Beispiel Kurve erneut verhandeln, nachdem die Lehrerin Edgars Nachfrage in Beispiel 2 verneint hatte. Nun bringt Piet die Kurve erneut in das Gespräch ein.

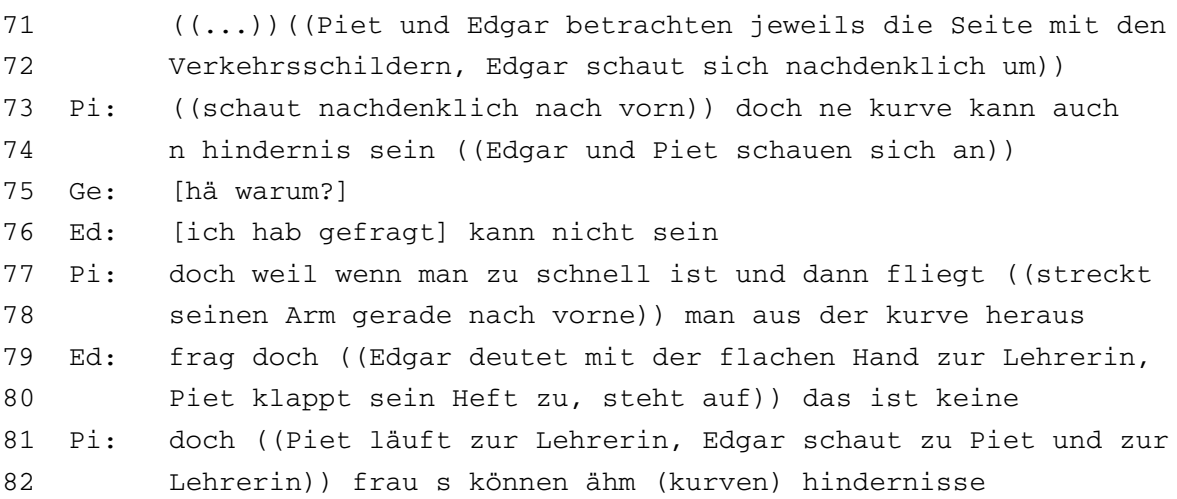




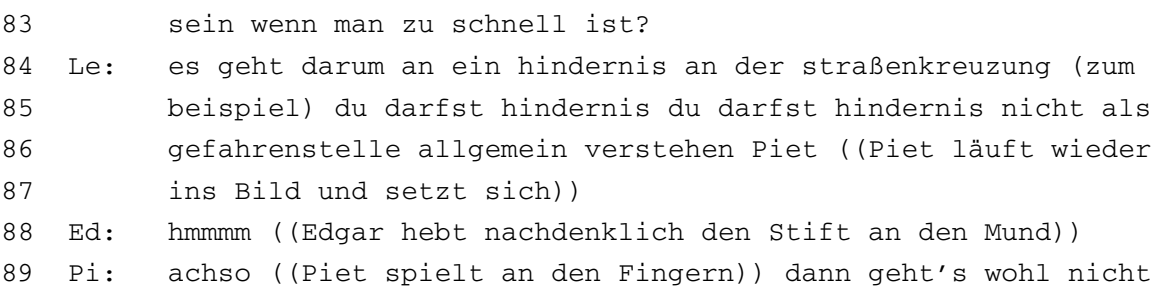

Piet leitet seinen Beitrag mit „doch“ ein und scheint Edgar damit widersprechen zu wollen. Parallel fragt Gerald nach einer Begründung (Z. 75) und Edgar führt an, dass dies nicht sein könne, da er gefragt habe (Z. 76). In Piets Argumentation in Zeile 77/78 wird deutlich, dass für ihn die Bedeutung Hindernis mit Gefahr verbunden ist, wenn ,man zu schnell“ ist. Implizit wird deutlich, dass eine Kurve - ähnlich wie (andere) Hindernisse - ein verändertes Verhalten von den Radfahrer*innen verlangt. Er begleitet seine Argumentation gestisch. Damit zeigt er an, was passiert, wenn man als Fahrradfahrer die Kurve nicht bekommt und verweist auf subjektives körperliches Erleben in dieser Situation. Edgars Logik ist eine andere - er verweist Piet auf die Lehrerin. Sie scheint für ihn die Instanz zu sein, die eine eindeutige Zuordnung einer bestimmten Verkehrssituation zur Kategorie Hindernis absichern kann. Obwohl Piet sich sicher zu sein scheint (,doch“), läuft er zur Lehrerin und fragt sie. Mit ihrer Antwort bezieht sie sich auf die Vorstellung des Jungen und verweist auf die im Kontext der Verkehrserziehung wichtige Unterscheidung, ,hindernis nicht als gefahrenstelle allgemein" zu verstehen. Sie erweitert somit auch das verwendete Begriffsinventar. Der Erläuterung der Lehrerin scheint Piet nun nichts mehr entgegenzusetzen zu haben (,,achso dann geht's wohl nicht"). Es wird aber nicht deutlich, ob Piet mit der Erläuterung der Lehrerin, dass Hindernisse nicht Gefahrenstellen allgemein seien, seine bisherige Vorstellung von Hindernis revidiert bzw. präzisiert. Seine Antwort „dann geht's wohl nicht“ (Z. 89) scheint sich eher auf den nun nicht mehr möglichen Eintrag ins Arbeitsheft zu beziehen. Unklar bleibt auch, wie die Erläuterung der Lehrerin bei Gerald und Edgar deren Vorstellung von Hindernis beeinflusst hat.

\section{Schlussfolgerungen}

Zunächst fassen wir zusammen, welche Bedeutung die Vorstellungen der Schüler*innen, die Äußerungen der Lehrkraft und das Material für den Ko-Konstruktionsprozess haben:

In Bezug auf das von Thomas (2015) und Pech (2009) (Abschn. 2.1) aufgezeigte Spannungsfeld des Faches Sachunterricht, verdeutlichen die Analysen, dass sich die fachlichen Aushandlungen zwischen den gesellschaftlichen Forderungen und den lebensweltlichen Bezügen der Kinder bewegen. In den Szenenabschnitten zeigt sich, wie die Kinder aus ihrer lebensweltlichen Erfahrung heraus, bestehend aus (körperlichem) Erleben (,,aus der Kurve fliegen“), aber auch aus Informationen, die sie eventuell auf anderen Wegen erfahren haben (,,bei der Karibik“), die unterschiedlichen Bedeutungen ko-konstruieren. Neben den Vorstellungen bzw. Präkonzepten 
zum Begriff Hindernis sind für diesen Prozess auch das Arbeitsmaterial und die Äußerungen der Lehrerin relevant.

Die Aufgabenstellung greift die Konzepte, die die Kinder von einem Hindernis haben, nicht auf, sondern setzt die für den Straßenverkehr relevante Bedeutung voraus. Die Jungen beginnen selbstständig einen Aushandlungsprozess über den Begriff bzw. die von ihnen vermuteten Bedeutungen. Die Abbildungen im Arbeitsmaterial sowie die zweite Fragestellung führen dazu, dass bestimmte Beispiele diskutiert und Begründungen angeführt werden. Die Lehrerin bietet den Jungen eine „Rückversicherung“ und bringt weitere Bedeutungsaspekte ein. In der Auseinandersetzung zur Frage danach, ob eine Kurve im Sinne der hier erfragten Bedeutung ein Hindernis sei, wird sichtbar, wie sich die Kinder an ihren eigenen Vorstellungen, an denen der anderen Kinder sowie an der von der StVO gesetzten und von der Lehrkraft vertretenen Vorstellung abarbeiten. Im Sinne der Conceptual Change Forschung werden die Präkonzepte der Kinder in dieser Auseinandersetzung auf den Prüfstand gestellt und verunsichert. Inwieweit sich in diesen Sequenzen bereits eine Veränderung in Richtung fachlich belastbarer Vorstellungen vollzieht, kann nicht eindeutig beantwortet werden.

Die Analyse zeigt, dass die Kinder ganz im Sinne der Sachunterrichtsdidaktik ihre Vorstellungen zum Fachbegriff Hindernis verhandelten, obwohl dies weder vom Unterrichtsmaterial noch von der Lehrkraft zunächst vorgesehen war. Auf diese Weise werden Lernprozesse angebahnt, im oben zitierten Sinne einer Weiterentwicklung der Schülervorstellungen, statt lediglich träges Wissen aufzubauen (Abschn. 2.3).

\section{Ausblick}

Die hier vorgestellten Analysen geben Hinweise auf eine Verortung der Fachlichkeit im inklusiven Sachunterricht, beziehen sich aber bisher nur auf einen Gruppentisch und einen kurzen Zeitabschnitt. In einem nächsten Schritt sollen die Aushandlungsprozesse an anderen Gruppentischen zu dieser Frage rekonstruktiv interaktionsanalytisch ausgewertet werden. Kontrastierende Analysen in Bezug auf die Wirkmächtigkeit des Arbeitsheftes können zeigen, wie sich die Aushandlung zum Begriff Hindernis zwischen einer Fokussierung auf die fachliche Auseinandersetzung und der korrekten Lösung der Aufgabe bewegen.

Bezüglich der Teilhabe aller kann sich folgende Analyse anschließen: An dem hier analysierten Gruppentisch sitzt ein vierter Junge (Sujan), der zu diesem Zeitpunkt erst seit einer Woche in der Klasse war. Es ist (fast) keine wechselseitige Interaktion der Jungen zu beobachten. Über sein Konzept zum Begriff Hindernis erfahren wir hier nichts, obwohl er über ein solches sicher auch verfügt, es aber in der ihm noch fremden Sprache Deutsch nicht mitteilt. Hier können Szenen im weiteren Unterrichtsverlauf in den Blick genommen werden, wenn er zunehmend mit seinen Mitschüler*innen interagiert.

Insgesamt zeigt die Analyse im Rahmen der Radfahrausbildung in einer heterogenen Lerngruppe, dass die Radfahrprüfung und auch die vorbereitenden Übungsmaterialien nicht zieldifferent angelegt sind, weil alle gleichermaßen auf diese Prüfung vorbereitet werden. Die analysierte Szene macht jedoch deutlich, wie unterschiedlich 
die Wege zu dieser Zielerreichung sein können, obwohl ein differenziertes Vorgehen und eine Verhandlung relevanter Vorstellungen nicht vorgesehen war. Nichtsdestotrotz bleibt die Frage, wie Schüler*innen Mobilität ermöglicht werden kann, die nicht mit dem undifferenzierten Material arbeiten können/die sich nicht mithilfe des Materials auf die Radfahrprüfung vorbereiten können. Dieser Aspekt einer ko-konstruktiven Verhandlung des Gegenstands im Sachunterricht unter Berücksichtigung der unterschiedlichen Vorstellungen und Beteiligungsmöglichkeiten der Kinder sowie der Fachlichkeit der Auseinandersetzung könnte in der weiteren Forschung noch stärker in den Blick genommen werden.

Funding Open Access funding enabled and organized by Projekt DEAL.

Open Access Dieser Artikel wird unter der Creative Commons Namensnennung 4.0 International Lizenz veröffentlicht, welche die Nutzung, Vervielfältigung, Bearbeitung, Verbreitung und Wiedergabe in jeglichem Medium und Format erlaubt, sofern Sie den/die ursprünglichen Autor(en) und die Quelle ordnungsgemäß nennen, einen Link zur Creative Commons Lizenz beifügen und angeben, ob Änderungen vorgenommen wurden.

Die in diesem Artikel enthaltenen Bilder und sonstiges Drittmaterial unterliegen ebenfalls der genannten Creative Commons Lizenz, sofern sich aus der Abbildungslegende nichts anderes ergibt. Sofern das betreffende Material nicht unter der genannten Creative Commons Lizenz steht und die betreffende Handlung nicht nach gesetzlichen Vorschriften erlaubt ist, ist für die oben aufgeführten Weiterverwendungen des Materials die Einwilligung des jeweiligen Rechteinhabers einzuholen.

Weitere Details zur Lizenz entnehmen Sie bitte der Lizenzinformation auf http://creativecommons.org/ licenses/by/4.0/deed.de.

\section{Literatur}

\section{Verwendete Literatur}

Adamina, M., Kübler, M., Kalcsics, K., Bietenhard, S., \& Engel, E. (2018). Vorstellungen von Schülerinnen und Schülern zu Themen des Sachunterrichts und des Fachbereichs Natur, Mensch, Gesellschaft - Einführung. In M. Adamina, M. Kübler, K. Kalcsics, S. Bietenhard \& E. Engel (Hrsg.), ,Wie ich mir das denke und vorstelle... ": Vorstellungen von Schülerinnen und Schülern zu Lerngegenständen des Sachunterrichts und des Fachbereichs Natur, Mensch, Gesellschaft. Bad Heilbrunn: Klinkhardt.

Baar, R., \& Maier, M. S. (2012). Was ist Familie? Die Grundschulzeitschrift, 26(252/253), 46-51.

Bräuer, C., Kunze, K., Pflugmacher, T., \& Rabenstein, K. (2018). Zur Konstruktion von Fachlichkeit. Eine Topologie am Beispiel von Forschung zu Literaturunterricht. In M. Martens, K. Rabenstein, K. Bräu, M. Fetzer, H. Gresch, I. Hardy \& C. Schelle (Hrsg.), Konstruktionen von Fachlichkeit. Ansätze, Erträge und Diskussionen in der empirischen Unterrichtsforschung (S. 111-124). Bad Heilbrunn: Klinkhardt.

Breidenstein, G. (2002). Interpretative Unterrichtsforschung - eine Zwischenbilanz und einige Zwischenfragen. In G. Breidenstein, A. Combe, W. Helsper \& B. Stelmaszyk (Hrsg.), Forum qualitative Schulforschung 2: Interpretative Unterrichts- und Schulbegleitforschung (S. 11-28). Wiesbaden: Springer.

Feuser, G. (2018). Thesen zur Integration - Inklusion im Sinne einer Allgemeinen Pädagogik. https:// www.georg-feuser.com/wp-content/uploads/2019/04/Feuser-Thesen-zur-Integration-Inklusion-i.S.Allgemeiner-P\%C3\%A4dagogik-Orig-1984-10-2018.pdf. Zugegriffen: 20. Mai 2020.

Funk, W., Hecht, P., Nebel, S., \& Stumpf, F. (2013). Verkehrserziehung in Kindergärten und Grundschulen. Berichte der Bundesanstalt für Straßenwesen. Mensch und Sicherheit, Heft M 238. Bremerhaven: Wirtschaftsverlag NW.

Gesellschaft für Didaktik des Sachunterrichts (GDSU) (2013). Perspektivrahmen. Bad Heilbrunn: Klinkhardt. 
Gropengießer, H. (2001). Didaktische Rekonstruktion des Sehens. Beiträge zur Didaktischen Rekonstruktion, Bd. 1. Oldenburg: Didaktisches Zentrum.

Günther, R., \& Degener, S. (2009). Psychomotorische Defizite von Kindern im Grundschulalter und ihre Auswirkungen auf die Radfahr-Ausbildung, Forschungsbericht VV 02, Gesamtverband der Deutschen Versicherungswirtschaft e. V., Berlin. https://www.verkehrswacht-medien-service.de/wpcontent/uploads/VMS-Studie-Psychomotorische-Defizite-und-Radfahrausbilung-2009.pdf. Zugegriffen: 10. März 2020.

Hackbarth, A., \& Martens, M. (2018). Inklusiver (Fach-)Unterricht: Befunde - Konzeptionen - Herausforderungen. In T. Sturm \& M. Wagner-Willi (Hrsg.), Handbuch schulische Inklusion (S. 191-206). Opladen: Barbara Budrich.

Hinz, A. (2011). Inklusive Pädagogik - Vision und konkretes Handlungsprogramm für den Sachunterricht? In H. Giest, A. Kaiser \& C. Schomaker (Hrsg.), Sachunterricht - auf dem Weg zur Inklusion (S. 354-361). Bad Heilbrunn: Klinkhardt.

Jonen, A., Möller, K., \& Hardy, I. (2003). Lernen als Veränderung von Konzepten - am Beispiel einer Untersuchung zum naturwissenschaftlichen Lernen in der Grundschule. In C. Diethard \& H.-J. Schwier (Hrsg.), Lernen und Aneignungsformen im Sachunterricht (S. 93-108). Bad Heilbrunn: Klinkhardt.

Kahlert, J. (2009). Der Sachunterricht und seine Didaktik. Bad Heilbrunn: Klinkhardt.

Kaiser, A. (2016). Neue Einführung in die Didaktik des Sachunterrichts. Baltmannsweiler: Schneider.

KMK (Sekretariat der Ständigen Konferenz der Kultusminister der Länder in der Bundesrepublik Deutschland) (2012). Empfehlung zur Mobilitäts- und Verkehrserziehung in der Schule. Beschluss der Kultusministerkonferenz vom 07.07.1972 i.d. F. vom 10.05.2012. Bonn: Sekretariat der KMK.

Köhnlein, W. (2015). Aufgaben und Ziele des Sachunterrichts. In J. Kahlert (Hrsg.), Handbuch Der Sachunterricht und seine Didaktik (S. 89-99). Bad Heilbrunn: Klinkhardt.

Krummheuer, G. (2012). Interaktionsanalyse. In F. Heinzel (Hrsg.), Methoden der Kindheitsforschung: Ein Überblick über Forschungszugänge zur kindlichen Perspektive (S. 234-247). Weinheim: Beltz Juventa.

Krummheuer, G., \& Brandt, B. (2001). Paraphrase und Traduktion: Partizipationstheoretische Elemente einer Interaktionstheorie des Mathematiklernens in der Grundschule. Weinheim: Beltz.

Krummheuer, G., \& Naujok, N. (1999). Grundlagen und Beispiele interpretativer Unterrichtsforschung. Opladen: Leske + Budrich.

Kucharz, D. (2015). Inklusiver Sachunterricht. In C. Huf \& I. Schnell (Hrsg.), Inklusive Bildung in Kita und Grundschule (S. 221-236). Stuttgart: Kohlhammer.

Möller, K. (1999). Konstruktivistisch orientierte Lehr-Lernprozessforschung im naturwissenschaftlichtechnischen Bereich des Sachunterrichts. In W. Köhnlein (Hrsg.), Vielperspektivisches Denken im Sachunterricht Forschungen zur Didaktik des Sachunterrichts (Bd. 3, S. 125-191). Bad Heilbrunn: Klinkhardt.

Möller, K. (2018). Die Bedeutung von Schülervorstellungen für das Lernen im Sachunterricht. In M. Adamina, M. Kübler, K. Kalcsics, S. Bietenhard \& E. Engel (Hrsg.), ,, Wie ich mir das denke und vorstelle... “: Vorstellungen von Schülerinnen und Schülern zu Lerngegenständen des Sachunterrichts und des Fachbereichs Natur, Mensch, Gesellschaft (S. 35-50). Bad Heilbrunn: Klinkhardt.

Pech, D., \& Rauterberg, M. (2016). Wozu Didaktik? Ein Beitrag zum Verhältnis von Sachunterrichtsdidaktik und Inklusion. In O. Musenberg \& J. Riegert (Hrsg.), Didaktik und Differenz (S. 134-114). Bad Heilbrunn: Klinkhardt.

Pech, D., Schomaker, C., \& Simon, T. (2018). Sachunterrichtsdidaktik \& Inklusion. Ein Beitrag zur Entwicklung. Hohengehren: Schneider.

Pech, D., Schomaker, C., \& Simon, T. (2019). Inklusion im Sachunterricht. Perspektiven der Forschung. Bad Heilbrunn: Klinkhardt.

Pech, D. (2009). Sachunterricht - Didaktik und Disziplin. Annäherungen an ein Sachlernverständnis im Kontext der Fachentwicklung des Sachunterrichts und seiner Didaktik. In www.widerstreit-sachunterricht.de, 13, S. 1-10. https://www.widerstreit-sachunterricht.de/ebeneI/didaktiker/pech/did_dis.pdf. Zugegriffen: 30. März 2020.

Schneider, L. V. (2018). Außerschulische Lernorte der Mobilitäts- und Verkehrserziehung im Sachunterricht: Lernprozesse im Spannungsfeld der Ziele und Interessen der Anbieter. In A. Flügel, M. Gröger, D. J. Schneider \& J. Wiesemann (Hrsg.), Außerschulische Lernorte von Kindern. Reflexionen - Konzeptionen-Perspektiven (S. 17-36). Siegen: Universitätsverlag.

Schomaker, C. (2007). Der Faszination begegnen. Ästhetische Zugangsweisen im Sachunterricht für alle Kinder. Oldenburg: Didaktisches Zentrum Carl-von-Ossietzky-Univ.

Seitz, S. (2005). Zeit für inklusiven Sachunterricht. Baltmannsweiler: Schneider. 
Simon, T. (2017). Vielperspektivität im Sachunterricht - Annäherungen an inklusionspädagogische und -didaktische Begründungslinien. In H. Giest, A. Hartinger \& S. Tänzer (Hrsg.), Vielperspektivität im Sachunterricht (S. 177-184). Bad Heilbrunn: Klinkhardt.

Spitta, P. (2015). Mobilitätsbildung. In J. Kahlert, M. Fölling-Albers, M. Götz, A. Hartinger, S. Miller \& S. Wittkowske (Hrsg.), Handbuch Didaktik des Sachunterrichts (S. 199-203). Bad Heilbrunn: Klinkhardt.

Thomas, B. (2015). Der Sachunterricht und seine Konzeptionen: historische und aktuelle Entwicklungen. Bad Heilbrunn: Klinkhardt.

Verkehrswacht. (2019). Kinder sicher im Verkehr. Mal- und Arbeitsbuch für die Radfahrausbildung. Detmold: K\&L.

\section{Internetquellen}

DWDS - Digitales Wörterbuch der deutschen Sprache, hrsg. v. d. Berlin-Brandenburgischen Akademie der Wissenschaften Hindernis. https://www.dwds.de/wb/Hindernis. Zugegriffen: 30. März 2020.

UN Behindertenrechtskonvention (UN-BRK). (2006). Übereinkommen über die Rechte von Menschen mit Behinderungen vom 13. Dezember 2006. https://www.behindertenrechtskonvention.info/. Zugegriffen: 30. März 2020.

Verkehrswacht Verkehrserziehung in der Sonderpädagogik. https://www.verkehrswacht-medien-service. de/grundschule/verkehrserziehung-gs/verkehrserziehung-in-der-sonderpaedagogik/. Zugegriffen: 30. März 2020. 